\title{
Restoring confidence among survivors of domestic abuse
}

Ann Marie Corrado

Faculty Reviewer: Lorraine Davies, PhD (Department of Sociology)

\section{INTRODUCTION}

Dr Mohammed Elahi earned his MD from the Schulich School of Medicine and Dentistry at Western University in 1993 and is now a Double Board Certified surgeon working in the Greater Toronto Area. He is one of three surgeons in Canada qualified in both Otolaryngology Head and Neck Surgery and Plastic and Reconstructive Surgery, and devotes time specifically to providing treatment to survivors of domestic abuse. In this interview, he explains how he became involved in this work and its importance. He describes the benefits of his treatment to his patients' physical, emotional and psychological well-being, as it is the first step in healing. Finally, he provides advice for medical students and other health care practitioners on how to do more to help women recover from the health effects of domestic violence, and thus re-establish their lives with confidence.

UWOMJ: Tell us a bit about yourself and how you came to be a plastic surgeon?

Mohammed Elahi: I grew up in the West End of Toronto and had a desire to help others from an early age. As a teenager, I helped take care of my grandparents who had health issues. These experiences with my own family members motivated me to pursue medical school at the University of Western Ontario and I am now a proud graduate of the class of 1993.

My main topic of interest during medical school was children with deformities, including cleft lip and cleft palate. I was exposed to these health conditions as a young teenager during my travels to Pakistan. Sadly, I learned that the majority of these children were marginalized in society and I became motivated to help these young people become more confident. I feel that my desire to improve their lives fueled my interest in plastic surgery, especially the reconstructive aspect of the specialty.

\section{What inspired you to begin caring for women who are vic- tims of abuse?}

My first introduction to women who have experienced domestic violence was a young woman who, as a consequence of being beaten and assaulted, had multiple facial fractures and lacerations. I discovered that she was being shuttled between many physicians, none of whom wanted to take on her care because of related health issues such as HIV status, hepatitis C, and drug addiction. She was transferred to my care during my first year of practice and I immediately took on her challenging case. The reconstruction demands in her case were quite difficult, but being responsible for her care and rehabilitation was very fulfilling. It was personally satisfying to see how grateful she was for the care she received and the extraordinary improvement in health and self-confidence that arose from the surgery I performed. It was clear that this reconstructive surgery affected her ability to make significant changes in her life. She gave up prostitution, stopped taking drugs, and in the process regained custody of her children. Remarkably, she still comes to see me every year to check in. It makes me so happy to see how she has transformed her life and become so confident. She reminds me why it is so important to support women who are struggling in every way one can.

What sorts of physical traumas and procedures are unique to women who have experienced domestic abuse?

From a plastic surgery perspective, the injuries I normally see are grouped in a few categories. First, there are lacerations to the face, arms, upper extremities, and lower extremities. I've also seen many stabbings over the course of my career. A second category that is common is facial fractures, including nasal fractures, mandibular fractures, orbital fractures, or a combination thereof. Furthermore, I see fractures in the upper extremity and hands. Finally, I frequently see burns that are a direct result of hot water and acid.

\section{What sorts of challenges do they face?}

In terms of challenges, there are multiple issues. To begin, women often experience many mental health issues because of the violence. It can be challenging finding care for the trauma that they have experienced. Many survivors benefit from psychiatric and psychological support in the acute phase of their injury and often in the long-term as well. Unfortunately, many of the patients I see have also experienced difficult childhoods with many hardships throughout their lives. They often confront systemic barriers, economic difficulties, and continuing violence. For those from a lower socioeconomic status, obtaining food, shelter, and clothing is a challenge. Unfortunately, these financial barriers sometimes constrain their ability to leave violent intimate partner relationships. Those who are mothers must also navigate joint custody, which elevates the risk of continuing abuse.

As a plastic surgeon my role is to reconstruct the external appearance. What I have learned is that the impact of surgery is greater than an improved physical appearance. The surgery is paramount in terms of starting the process of healing and regaining confidence. I have also learned, however, that the health effects of domestic abuse are multiple, deep, and often long-term. Survivors navigate complicated lives with issues that require attention and support over the long run. The inner wounds are definitely more severe than is generally recognized. That is why I refer all my patients to other health professionals so that they can get the care they deserve and require.

\section{How does mental health play a role in recovery?}

Mental health plays a significant role in women who have suffered from abuse. Whenever I see a woman who was a victim of domestic violence, I have three allied health professionals involved 
from the beginning. This includes a psychiatrist for acute psychiatric issues, a psychologist for coping issues, and a social worker to enhance their well-being. These allied health professionals are truly incredible in helping survivors gain access to all the resources they require to maintain their affairs successfully. The management of domestic abuse patients truly requires a multi-disciplinary approach.

\section{WHAT I HAVE LEARNED IS THAT THE}

IMPACT OF SURGERY IS GREATER THAN

AN IMPROVED PHYSICAL APPEARANCE.

\section{THE SURGERY IS PARAMOUNT IN}

TERMS OF STARTING THE PROCESS OF

HEALING AND REGAINING CONFIDENCE.

\section{Can you explain the reactions from women after they have} healed from surgery?

As a plastic surgeon, seeing women's response to the surgery is extremely satisfying. I cannot tell you the number of times I've seen women cry and the number of times I have been hugged because they were so thankful. To see the look on their face when the stitches and bandages come off after the reconstruction is powerful. I feel strongly that it is the first step in healing the trauma they endured and in re-establishing their lives with a clean slate.

\section{How has helping these women changed your outlook or prac-} tice?

My medical training at the University of Western Ontario taught me the importance of always being an advocate for the most disadvantaged individuals in society. I truly believe that this is my obligation as a doctor and it is also part of the Hippocratic oath. The fact that other physicians did not treat the first woman I mentioned really disappointed me. No one deserves to be judged based on their socioeconomic status, their past medical history, and the circumstances of their plight. I certainly will continue caring for these women for the rest of my career as this work is extremely rewarding and meaningful.

\section{What financial considerations are involved in helping these pa- tients?}

Although the health care system in Canada gets criticized a lot, I think it is a wonderful system. It covers all the reconstructive elements for women who are victims of abuse. In terms of the secondary cosmetic procedures, I provide them free of charge at my own cost and I am happy to do so. Again, I feel that it is something extremely small that I can do. It makes a world of a difference and can really aid these women in the healing process.

What advice would you give other physicians or healthcare professionals, who want to dedicate their time and skills to helping women who have experienced abuse?
I believe that all plastic surgeons, physicians, allied healthcare professionals and fellow citizens have an ethical obligation to help and assist others. As a doctor and healthcare provider, you are blessed with the ability to fix and repair the physical traumas that people sustain. To be able to help the most vulnerable individuals is so satisfying. These are people who are often forgotten and neglected and they might not feel worthy of your time or what you are investing in them. However, it is important that you make them feel safe, cared for, and supported. We all have a mother and many of us have sisters, wives, and daughters. On behalf of all women, it is imperative that we assist them in any way we can. 